PSS PROCEEDINGS

\title{
Status of Higher Order QCD calculations
}

\section{A. Gehrmann-De Ridder*}

Institute for theoretical Physics, ETH-Zurich, CH-8093 Zürich

E-mail: gehraeitp.phys.ethz.ch

In this talk, we review recent developments in fixed order perturbative calculations for observables at high energy colliders.

35th International Conference of High Energy Physics - ICHEP2010,

July 22-28, 2010

Paris France

${ }^{*}$ Speaker. 


\section{Introduction}

At high energy colliders, the interaction between elementary matter particles (quarks and gluons), is dominated by the strong force which is very well described by the theory of QCD. As protons are not elementary particles, but composed of quarks and gluons, and as most physics observables studied at the LHC involve jets in the final state, QCD effects are omnipresent in hadronic collisions. A detailed understanding of QCD is therefore mandatory for the interpretation of collider data. At large momentum transfer, perturbative expansion of QCD can be used to obtain quantitative predictions for hadronic observables.In this talk, we review recent progress on perturbative QCD calculations for two categories of observables: multiparticle production at NLO and precision observables at NNLO.

\section{Multiparticle Production at NLO}

The search for new physics signals at the CERN LHC will often involve multi-particle final states, consisting of numerous jets, leptons, photons and missing energy. Quite in general, at LHC one expects the production of massive short-lived particles which will be detected through their decay signatures. Meaningful searches for these signatures require not only a very good anticipation of the expected signal, but also of all standard model backgrounds yielding identical final state signatures. Since leading-order calculations are affected by large uncertainties in their normalization and their kinematical dependence, it appears almost mandatory to include NLO corrections.

An NLO calculation of a $n$-particle observable consists of two contributions: the virtual oneloop correction to the $n$-particle production process, and the real radiation contribution from the $(n+1)$-particle production process. Both contributions are infrared divergent, and can be evaluated numerically only after extracting the infrared divergent contributions from the real radiation process. Restricting ourselves to processes involving massless final states, several well-established and widely used methods exist for this task [1]. The evaluation of the one-loop multi-leg amplitudes poses a challenge in complexity and stability. Any one-loop amplitude can be expressed as a linear combination of one-loop integrals with at most four external legs, plus a rational remainder. Enormous progress has been made in recent years in the systematic computation of the oneloop integral coefficients and rational terms. While previously established Feynman-diagram based techniques for tensor reduction and form factor decomposition were successfully extended [2] to multi-leg problems, a new arsenal of techniques was emerging from the use of unitarity and multiparticle cuts. Using these, the one-loop integral coefficients of an amplitude can be inferred without evaluation of all individual diagrams [3]. The rational coefficients can be determined in the same framework by extending the unitarity relations from four dimensions to higher-dimensional spacetime [4].

Given the large number of different multi-particle final states of potential interest to new physics searches, an automation of NLO calculations is highly desirable. The implementation of the real radiation contributions and their infrared subtraction terms has been accomplished in the Sherpa [5], MadGraph [6] and Helac/Phegas [7] frameworks. while the automation of the virtual corrections has been achieved using the various available methods. A semi-numerical form factor decomposition is automated in the Golem package [8]. Unitarity and multi-particle cuts are 
used in the BlackHat package [9], and the OPP method is automated in CutTools [10]. Numerical $D$-dimensional unitarity is applied in the Rocket package [11] and the Samurai package [12];

The current frontier of complexity are NLO calculations of $2 \rightarrow 4$ reactions. Several very important processes of this type have been computed most recently. The QCD background process to the associated production of a Higgs with a heavy quark-antiquark pair yielding a $t \bar{t} b \bar{b}$ final states were computed recently to NLO [13] A detailed overview on the status of this calculation will be given by S. Dittmaier, at this conference [14].

NLO corrections of $W+3 j$ were obtained by two groups in the Rocket [15] and in the Blackhat+Sherpa [16] framework. The corrections to $Z^{0}+3 j$ were also obtained with Blackhat+Sherpa [17]. The results will be reported by D. Kosower, during this conference [18].

\section{Precision Observables at NNLO}

Few benchmark observables (e.g. jet cross sections, vector boson production) are measured experimentally to an accuracy of one per cent or below. For a theoretical interpretation of these observables, NNLO corrections are mandatory. Likewise, NNLO corrections are required for a reliable description of observables with potentially large perturbative corrections, like Higgs or vector boson production.

The calculation of NNLO corrections to an $n$-particle final state requires three ingredients: the two-loop matrix elements for the $n$-particle production, the one-loop matrix elements for the $(n+$ 1)-particle production and the tree-level matrix elements for $(n+2)$-particle production. The latter two contributions develop infrared singularities if one or two particles become soft or collinear, requiring a subtraction method to extract these infrared poles, which are then combined with the virtual corrections to yield a finite prediction. The two major challenges of NNLO calculations are the two-loop matrix elements and the handling of the real radiation at NNLO. Up to now, two types of approaches to real radiation have been applied in NNLO calculations of exclusive observables. The sector decomposition method [19] is based on a systematic expansion in distributions, followed by numerical integration over many different small phase space sectors. Subtraction methods search to approximate the full real radiation contribution by subtraction terms in all unresolved limits; these terms are then integrated analytically. While many subtraction methods have been worked out at NLO, only two methods have so far yielded results at NNLO: the antenna subtraction method [20] for processes in $e^{+} e^{-}$annihilation, and the $q_{T}$-subtraction [21] for hadron collider processes in specific kinematic configurations.

For the purpose of these proceedings contributions, we restrict ourselves to the description of two particular observables: Vector boson and jet production. Fully exclusive NNLO corrections to vector boson production have been derived using sector decomposition [22] and with $q_{T^{-}}$ subtraction [23], including the leptonic vector boson decay. Using the newly obtained results, the NNLO corrections (and their uncertainty) to the lepton charge asymmetry [24] can be quantified.

Jet production observables have been computed to NNLO only for $e^{+} e^{-}$annihilation up to now. Two implementations of the NNLO corrections to $e^{+} e^{-} \rightarrow 3 j$ and related observables are available [25] both based on antenna subtraction. Including these new NNLO corrections, LEP data on event shapes and jet cross sections were reanalyzed in view of an improved determination of the strong coupling constant [26]. 
The derivation of NNLO corrections to jet cross sections in hadronic collisions is of high priority. The relevant two-loop matrix elements for hadronic collisions and for deep inelastic scattering [27] are known for some time already, and substantial progress is being made to extend the antenna subtraction method to include hadrons in the initial state. A major step towards this aim has been achieved [28] where the subprocess to hadronic dijet production $g g \rightarrow 4 g$ has been evaluated in this antenna framework. The integrated forms of all antenna functions have been derived for one parton in the initial state [29], the case of two initial state partons [30] is work in progress.

\section{Conclusions}

In this talk we outline most recent progresses for calculation of higher order corrections to observables in hadronic collisions. We presented the enormous progress on NLO calculations for multi-leg final states and outlined first NNLO results for fully differentiable observables.

\section{Acknowledgments}

The author gratefully ackowledges the support by the Swiss National Science Foundation (SNF) under contract PP0022-118864 and in part by the European Commission through the 'LHCPhenoNet' Initial Training Network PITN-GA-2010-264564'.

\section{References}

[1] S. Frixione, Z. Kunszt and A. Signer, Nucl. Phys. B 467 (1996) 399; S. Catani and M. H. Seymour, Nucl. Phys. B 485 (1997) 291;A. Gehrmann-De Ridder, T. Gehrmann and E. W. N. Glover, JHEP 0509 (2005) 056; A. Daleo, T. Gehrmann and D. Maître, JHEP 0704 (2007) 016.

[2] A. Denner and S. Dittmaier, Nucl. Phys. B 734 (2006) 62; T. Binoth, J. P. Guillet, G. Heinrich, E. Pilon and C. Schubert, JHEP 0510 (2005) 015.

[3] G. Ossola, C. G. Papadopoulos and R. Pittau, JHEP 0805 (2008) 004 [0802.1876].

[4] R. K. Ellis, W. T. Giele, Z. Kunszt and K. Melnikov, Nucl. Phys. B 822 (2009) 270 [0806.3467].

[5] T. Gleisberg and F. Krauss, Eur. Phys. J. C 53 (2008) 501 [0709.2881].

[6] R. Frederix, T. Gehrmann and N. Greiner, JHEP 0809 (2008) 122 [0808.2128].

[7] M. Czakon, C. G. Papadopoulos and M. Worek, JHEP 0908 (2009) 085 [0905.0883].

[8] T. Binoth, J. P. Guillet, G. Heinrich, E. Pilon and T. Reiter, Comput. Phys. Commun. 180 (2009) 2317.

[9] C. F. Berger et al., Phys. Rev. D 78 (2008) 036003 [0803.4180].

[10] G. Ossola, C. G. Papadopoulos and R. Pittau, JHEP 0803 (2008) 042 [0711.3596].

[11] W. T. Giele and G. Zanderighi, JHEP 0806 (2008) 038 [0805.2152].

[12] P. Mastrolia, G. Ossola, T. Reiter and F. Tramontano, [1006.0710].

[13] A. Bredenstein, A. Denner, S. Dittmaier and S. Pozzorini, JHEP 1003 (2010) 021; G. Bevilacqua, M. Czakon, C. G. Papadopoulos, R. Pittau and M. Worek, JHEP 0909 (2009) 109.

[14] S. Dittmaier, these proceedings. 
[15] R. Keith Ellis, K. Melnikov and G. Zanderighi, Phys. Rev. D 80 (2009) 094002 [0906.1445].

[16] C. F. Berger et al., Phys. Rev. D 80 (2009) 074036 [0907.1984].

[17] C. F. Berger et al., 1004.1659.

[18] D. Kosower, these proceedings.

[19] C. Anastasiou, K. Melnikov and F. Petriello, Phys. Rev. D 69 (2004) 076010; T. Binoth and G. Heinrich, Nucl. Phys. B 693 (2004) 134.

[20] A. Gehrmann-De Ridder, T. Gehrmann and E. W. N. Glover, JHEP 0509 (2005) 056.

[21] S. Catani and M. Grazzini, Phys. Rev. Lett. 98 (2007) 222002 [hep-ph/0703012].

[22] K. Melnikov and F. Petriello, Phys. Rev. D 74 (2006) 114017 [hep-ph/0609070].

[23] S. Catani, L. Cieri, G. Ferrera, D. de Florian and M. Grazzini, Phys. Rev. Lett. 103 (2009) 082001.

[24] S. Catani, G. Ferrera and M. Grazzini, JHEP 1005 (2010) 006 [1002.3115].

[25] A. Gehrmann-De Ridder, T. Gehrmann, E. W. N. Glover and G. Heinrich, JHEP 0711 (2007) 058, S. Weinzierl, JHEP 0907 (2009) 009 [0904.1145].

[26] G. Dissertori, A. Gehrmann-De Ridder, T. Gehrmann, E. W. N. Glover, G. Heinrich and H. Stenzel, Phys. Rev. Lett. 104 (2010) 072002 [0910.4283].

[27] T. Gehrmann and E. W. N. Glover, Phys. Lett. B 676 (2009) 146 [0904.2665].

[28] E. W. N. Glover and J. Pires, JHEP 1006 (2010) 096 [1003.2824].

[29] A. Daleo, A. Gehrmann-De Ridder, T. Gehrmann and G. Luisoni, JHEP 1001 (2010) 118.

[30] R. Boughezal, A. Gehrmann-De Ridder and M. Ritzmann, [1011.6631]. 